\title{
Inhalt
}

\section{§ 1 Einleitung - 1}

1. Inhaltlicher Fokus des Werkes - 1

2. Zur Arbeitsweise mit diesem Werk — 2

\section{$\S 2$ Lern- und Recherchetipps - 4}

1. Nach den schriftlichen Prüfungen -4

2. Aktuelles Tagesgeschehen und Fachzeitschriften - 4

3. Vorbereitung auf die jeweiligen Prüfer $-\mathbf{5}$

\section{§ 3 Rechtsgeschichte - 7}

1. Entstehungsgeschichte des $B G B-7$

2. Die Geschichte des Strafrechts - 9

3. Die Geschichte der Staatsanwaltschaft $\mathbf{- 1 0}$

4. Das Grundgesetz - $\mathbf{1 1}$

a. Entstehungsgeschichte - 11

b. Wesentliche Ereignisse und Änderungen des GG nach seinem Inkrafttreten - 12

c. Wichtige Unterschiede im Vergleich zur WRV - 13

5. Der Prozess der Europäischen Integration - $\mathbf{1 5}$

a. Wichtige Verträge und Vertragsreformen -15

b. Erweiterungsrunden der EU, Austritte - 16

c. Wesentliche Änderungen durch den Vertrag von Lissabon - 16 aa. Auflösung des Drei-Säulen-Modells - 16

bb. Verfahrensänderungen -17

cc. Ausweitung der Rechtsetzungskompetenzen - 17

dd. Direkte Demokratie — 17

ee. Verbindlichkeit der Grundrechtscharta - 17

ff. Neue Ämter - $\mathbf{1 8}$

gg. Freiwilliger Austritt aus der Union - 18

hh. EU als Völkerrechtssubjekt - 18

d. Das Kooperationsverhältnis zwischen dem BVerfG und dem EuGH -19

6. Wichtige Gesetze und deren Inkrafttreten - 22

7. Häufige Fragen aus dem Bereich Rechtsgeschichte $\mathbf{- 2 2}$

a. Zivilrecht $-\mathbf{2 2}$

b. Strafrecht -25 
c. Öffentliches Recht -27

d. Berühmte Juristen und sonstige Personen - $\mathbf{3 0}$

§4 Die Instanzenzüge - 35

1. Instanzenzug und Gerichtszuständigkeit im Zivilprozess - $\mathbf{3 5}$

2. Instanzenzug und Gerichtszuständigkeit im Strafprozess - $\mathbf{3 7}$

3. Instanzenzug und Gerichtszuständigkeit im Verwaltungsprozess $-\mathbf{3 8}$

$\S 5$ Wichtige Gerichte - 39

1. Das BVerfG -39

2. Das BVerwG $-\mathbf{4 0}$

3. Der $\mathrm{BGH}-\mathbf{4 0}$

4. Das BAG -41

5. Der Gemeinsame Senat der obersten Gerichtshöfe des Bundes - 41

6. Der EuGH -41

7. Der EGMR - $\mathbf{4 2}$

8. Die Landesverfassungsgerichtshöfe -42

§6 Verfahrensgrundsätze - 44

1. Verfahrensgrundsätze des Zivilprozesses - 44 Überblick — 44

a. Dispositionsmaxime $-\mathbf{4 4}$

b. Verhandlungsgrundsatz/Beibringungsgrundsatz -45

c. Beschleunigungsgrundsatz $-\mathbf{4 5}$

d. Rechtsstaatsprinzip: Rechtliches Gehör, faires Verfahren, Waffengleichheit - 45

e. Grundsatz der Mündlichkeit - 46

f. Grundsatz der Unmittelbarkeit - 46

g. Grundsatz der Öffentlichkeit -46

h. Grundsatz der Einheit der mündlichen Verhandlung — 46

2. Verfahrensgrundsätze des Strafprozesses — 47 Überblick - 47

a. Das Offizialprinzip - 47

b. Das Akkusationsprinzip - 48

c. Das Legalitätsprinzip - $\mathbf{4 8}$

d. Der Untersuchungsgrundsatz -48

e. Das Beschleunigungsgebot und die Konzentrationsmaxime $-\mathbf{4 8}$ 
f. Freie richterliche Beweiswürdigung — 49

g. Das Mündlichkeitsprinzip - 49

h. Der Unmittelbarkeitsgrundsatz $-\mathbf{5 0}$

i. Der Öffentlichkeitsgrundsatz $-\mathbf{5 0}$

j. In dubio pro reo $-\mathbf{5 0}$

k. Sonstige aus dem Rechtsstaatsprinzip folgende Grundsätze — $\mathbf{5 1}$

3. Verfahrensgrundsätze des Verwaltungsprozesses - $\mathbf{5 1}$

a. Untersuchungsgrundsatz $-\mathbf{5 1}$

b. Dispositionsmaxime $-\mathbf{5 2}$

c. Grundsatz der Mündlichkeit - $\mathbf{5 2}$

d. Beschleunigungsgrundsatz $-\mathbf{5 2}$

e. Weiteres $-\mathbf{5 3}$

§ 7 Verfassungsrechtliche Grundentscheidungen und Staatszielbestimmungen des GG -54

1. Republik $-\mathbf{5 4}$

2. Sozialstaat $-\mathbf{5 4}$

3. Bundesstaat $-\mathbf{5 4}$

4. Grundsatz des bundesfreundlichen Verhaltens - $\mathbf{5 5}$

5. Das Demokratieprinzip - $\mathbf{5 5}$

6. Umweltschutz $-\mathbf{5 6}$

7. Europäische Integration, Art. $23 \mathrm{GG}-56$

8. Rechtsstaatlichkeit $-\mathbf{5 7}$

a. Gewaltenteilung $-\mathbf{5 7}$

b. Gesetzmäßigkeit der Verwaltung - $\mathbf{5 8}$

c. Gesetzesvorbehalt $-\mathbf{5 8}$

d. Wesentlichkeitstheorie - $\mathbf{5 8}$

e. Rückwirkungsverbot $-\mathbf{5 9}$

f. Bestimmtheitsgebot $-\mathbf{5 9}$

§ 8 Häufige Fragen zu den drei Rechtsgebieten — 61

1. Zivilrecht -61

2. Strafrecht -68

3. Öffentliches Recht $-\mathbf{8 0}$

\section{§ 9 Leiturteile -94}

1. Leiturteile des EuGH $-\mathbf{9 4}$

2. Leiturteile des BVerfG — 98 
3. Leiturteile im Zivilrecht (RG und $B G H)-103$

4. Leiturteile im Strafrecht (Preuß. Obertribunal, RG und BGH) 108

§ 10 Fragen zu Abläufen in der Praxis - 113

1. Praxisfragen aus Sicht eines Rechtsanwalts - $\mathbf{1 1 3}$

2. Praxisfragen aus Sicht eines Richters — $\mathbf{1 1 7}$

3. Praxisfragen aus Sicht eines Staatsanwalts $-\mathbf{1 2 2}$

\section{§ 11 Juristische Methodik — 125}

1. Sauberes Arbeiten am Beispiel einer Analogie - $\mathbf{1 2 5}$

2. Professionelle Argumentation und Streitdarstellung - $\mathbf{1 2 5}$

3. Logische Fehler $\mathbf{1 2 6}$

4. Richtig Abwägen $-\mathbf{1 2 7}$

5. Die Auslegungscanones $-\mathbf{1 2 9}$

a. Wortlautauslegung $\mathbf{1 2 9}$

b. Systematische Auslegung — 129

c. Teleologische Auslegung - $\mathbf{1 3 0}$

d. Genetische und historische Auslegung — 130

e. Sonstiges zur Auslegung — 131

\section{$\S 12$ Liste lateinischer Begriffe und Sentenzen - 132}

\section{§ 13 Der Vortrag - 136}

1. Vorgaben der verschiedenen Bundesländer — $\mathbf{1 3 6}$

2. Bedeutung des Kurzvortrags in der ersten juristischen Prüfung - 136

3. Organisatorischer Ablauf der mündlichen Prüfung — 137

a. Das Vorgespräch — $\mathbf{1 3 7}$

b. Die Vorbereitung des Vortrags - 138

c. Die Präsentation des Vortrags — 139

d. Die Prüfungsgespräche -139

e. Die Notenbekanntgabe 139

4. Vorgaben des Justizprüfungsamtes an den Vortrag (Beispiel NRW) 140

5. Inhaltliche Bandbreite an Fragestellungen beim Vortrag - $\mathbf{1 4 2}$

a. Themenbezogene Fragen - $\mathbf{1 4 2}$

b. Das aktuelle Tagesgeschehen - 142

6. Rhetorik und Präsentationstechnik -142

a. Auditive Aspekte — $\mathbf{1 4 3}$

aa. Angemessene Artikulation — 143 
bb. Sachlicher Stil 143

cc. Stereotype Formulierung - 143

dd. Kurze Sätze — 144

ee. Deutliches Sprechen - 144

ff. Angemessene Sprechgeschwindigkeit — 144

gg. Freie Rede $-\mathbf{1 4 4}$

b. Visuelle Aspekte — $\mathbf{1 4 5}$

aa. Körperhaltung - 145

bb. Blickkontakt - 145

cc. Gestik 145

dd. Wohin mit den Händen? 146

ee. Mimik $\mathbf{1 4 6}$

c. Inhaltliche Präsentationsfragen - 146

aa. Überflüssiges weglassen - 146

bb. Genaue Gesetzesangaben — 146

cc. Keine Querverweise im Vortrag — 147

dd. Fremdwörter — 147

ee. Schwerpunktsetzung — 147

d. Aufregung bewältigen - 147

aa. Prüfungsangst $\mathbf{1 4 8}$

bb. Zurechtlegen von Standardformulierungen — 148

cc. Den Faden souverän verlieren - $\mathbf{1 4 8}$

7. Juristische Argumentation — $\mathbf{1 4 9}$

a. Juristische Argumentationsmuster 149

b. Sauberer Gutachtenstil - $\mathbf{1 4 9}$

c. Besonderheiten bei thematischen Aufgabenstellungen - 150

8. Die einstündige Vorbereitungsphase — $\mathbf{1 5 1}$

a. Zeiteinteilung — $\mathbf{1 5 1}$

aa. Grobe Zeitabschnitte - 151

bb. Eigene Stoppuhr? - 152

cc. Nicht die Zeit überschreiten! - 152

b. Lesen des Sachverhalts - $\mathbf{1 5 2}$

c. Einleitungssatz $-\mathbf{1 5 2}$

d. Die Lösungsskizze — $\mathbf{1 5 3}$

e. Die geistige Generalprobe $-\mathbf{1 5 3}$

9. Der Aufbau des Vortrags — 154

a. Begrüßung - 154 
b. Hauptteil - 154

c. Ende des Vortrags - $\mathbf{1 5 5}$

10. Vorbereitung auf den Kurzvortrag - 155

a. Repetieren des materiellen/prozessualen Rechts - $\mathbf{1 5 5}$

b. Training von Kurzvorträgen - 156

aa. Prüfungssituation möglichst wie im Examen - 156

bb. Regeln für die Lerngruppe - 156

cc. Weitere hilfreiche Trainingsmethoden -157

dd. Vorträge anschauen - 157

11. Fachspezifische Besonderheiten - 157

a. Besonderes bei Vorträgen im Zivilrecht - $\mathbf{1 5 7}$

b. Besonderes bei Vorträgen im Strafrecht - $\mathbf{1 5 8}$

c. Besonderes bei Vorträgen im öffentlichen Recht - 158

\section{§ 14 Übungsvorträge - 159}

1. Mustervortrag Zivilrecht -159

a. Sachverhalt $-\mathbf{1 5 9}$

b. Lösungsskizze -159

2. Mustervortrag Strafrecht -167

a. Sachverhalt - 167

b. Lösungsskizze -167

3. Mustervortrag Öffentliches Recht $-\mathbf{1 7 6}$

a. Sachverhalt - $\mathbf{1 7 6}$

b. Lösungsskizze - 177 